\title{
MULTI-POLYLOGS AT TWELFTH ROOTS OF UNITY AND SPECIAL VALUES OF WITTEN MULTIPLE ZETA FUNCTION ATTACHED TO THE EXCEPTIONAL LIE ALGEBRA $\mathfrak{g}_{2}$
}

\author{
JIANQIANG ZHAO
}

\begin{abstract}
Department of Mathematics, Eckerd College, St. Petersburg, Fl 33711, UsA
Max-Planck Institut für Mathematik, Vivatsgasse 7, 53111 Bonn, Germany
\end{abstract}

\begin{abstract}
In this note we shall study the Witten multiple zeta function associated to the exceptional Lie algebra $\mathfrak{g}_{2}$. Our main result shows that its special values at nonnegative integers can always be expressed as rational linear combinations of the multi-polylogs evaluated at 12th roots of unity, except for two irregular cases.
\end{abstract}

\section{INTRODUCTION AND PRELIMINARIES}

In [8] Witten related the volumes of the moduli spaces of representations of the fundamental groups of two dimensional surfaces to the special values of the following zeta function attached to complex semisimple Lie algebras $\mathfrak{g}$ at positive integers:

$$
\zeta_{W}(s ; \mathfrak{g})=\sum_{\varphi} \frac{1}{(\operatorname{dim} \varphi)^{s}}
$$

where $\varphi$ runs over all finite dimensional irreducible representations of $\mathfrak{g}$. Matsumoto and his collaborators recently defined the multiple variable analogs of $\zeta_{W}(s ; \mathfrak{g})$ and studied some of their analytical and arithmetical properties (see [4, 5, 6]). Let $\Delta_{+}$be the set of all positive roots of $\mathfrak{g}$ and $\left\{\lambda_{1}, \ldots, \lambda_{d}\right\}$ its fundamental weights. Then one can define (see [4, (1.6)]) the multiple zeta function attached to $\mathfrak{g}$ by setting

$$
\zeta_{\mathfrak{g}}\left(\left\{s_{\alpha}\right\}_{\alpha \in \Delta_{+}}\right):=\sum_{m_{1}, \ldots, m_{d}=1}^{\infty} \prod_{\alpha \in \Delta_{+}}\left\langle\alpha^{\vee}, m_{1} \lambda_{1}+\cdots+m_{d} \lambda_{d}\right\rangle^{-s_{\alpha}},
$$

where $\alpha^{\vee}$ is the coroot of $\alpha$. The relation to the original (single variable) Witten zeta function $\zeta_{W}(s ; \mathfrak{g})$ is given by the identity $((1.4)$ of loc. cit.)

$$
\zeta_{W}(s ; \mathfrak{g})=\left(\prod_{\alpha \in \Delta_{+}}\left\langle\alpha^{\vee}, \lambda_{1}+\cdots+\lambda_{d}\right\rangle\right)^{s} \cdot \zeta_{\mathfrak{g}}(s, \ldots, s) .
$$

In [13] and [14] we considered the special values of the Witten multiple zeta function attached to $\mathfrak{s o}(5)$ and $\mathfrak{s l}(4)$, respectively. The main results state that the special values of $\zeta_{\mathfrak{s} l(4)}$

1991 Mathematics Subject Classification. Primary: 11M41; Secondary: 40B05.

Key words and phrases. Witten multiple zeta function, multi-polylog. 
$\left(\right.$ resp. $\left.\zeta_{\mathfrak{s o}(5)}\right)$ at nonnegative integers can be expressed as $\mathbb{Q}$-linear combinations of multiple zeta values (44) (resp. alternating Euler sums, i.e., multi-polylogs (3) evaluated at \pm 1 ). For general Lie algebras we expect other special values of multi-polylogs at roots of unity should appear.

In this note we turn to the exceptional Lie algebra $\mathfrak{g}_{2}$. Let $\alpha_{1}$ and $\alpha_{2}$ be the two fundamental roots of $\mathfrak{g}_{2}$. Then the set of the positive roots is (see [2, p. 220])

$$
\Delta_{+}=\left\{\alpha_{1}, \alpha_{2}, \alpha_{1}+\alpha_{2}, \alpha_{1}+2 \alpha_{2}, \alpha_{1}+3 \alpha_{2}, 2 \alpha_{1}+3 \alpha_{2}\right\}
$$

Thus by definition (1)

$$
\zeta_{\mathfrak{g}_{2}}\left(s_{1}, \ldots, s_{6}\right)=\sum_{m, n=1}^{\infty} \frac{1}{m^{s_{1}} n^{s_{2}}(m+n)^{s_{3}}(m+2 n)^{s_{4}}(m+3 n)^{s_{5}}(2 m+3 n)^{s_{6}}} .
$$

To study the special values of $\zeta_{\mathfrak{g}_{2}}$ at nonnegative integers we need to utilize the multi-polylog function defined by

$$
L i_{s_{1}, \ldots, s_{d}}\left(x_{1}, \ldots, x_{d}\right):=\sum_{k_{1}>k_{2}>\cdots>k_{d}>0} \frac{x_{1}^{k_{1}} x_{2}^{k_{2}} \ldots x_{d}^{k_{d}}}{k_{1}^{s_{1}} k_{2}^{s_{2}} \ldots k_{d}^{s_{d}}}
$$

where $\left|x_{1} \cdots x_{j}\right|<1$ and $s_{j} \in \mathbb{N}$ for all $j$. We call $w:=s_{1}+\cdots+s_{d}$ the weight and $d$ the depth. The multi-polylog function can be continued meromorphically to a multi-valued function on $\mathbb{C}^{d}$ (see [12]). In particular we can consider its values at roots of unity. When $x_{j}=1$ for all $j$ we obtain the well-known multiple zeta values

$$
\zeta\left(s_{1}, \ldots, s_{d}\right):=\sum_{k_{1}>\cdots>k_{d}>0} \frac{1}{k_{1}^{s_{1}} \cdots k_{d}^{s_{d}}} .
$$

If all $x_{j}$ are $N$-th root of unity for some positive integer $N$ then we say the special value given by (3) has level $N$. In this case we have

$$
L i_{s_{1}, \ldots, s_{d}}\left(x_{1}, \ldots, x_{d}\right) \text { converges } \Longleftrightarrow\left(s_{1}, x_{1}\right) \neq(1,1)
$$

Let $\mathcal{M P V}(w, d, N)$ be the $\mathbb{Q}$-vector space generated by the the multi-polylog values of weight $w$, depth $d$ and level $N$. The main result of this note is the following

Theorem 1.1. Let $s_{1}, \ldots, s_{6}$ be nonnegative integers. Let $w=s_{1}+\cdots+s_{6}$. Then $\zeta_{\mathfrak{g}_{2}}\left(s_{1}, \ldots, s_{6}\right)$ converges if and only if

$$
w-s_{1}>1, w-s_{2}>1 \text {, and } w>2 \text {. }
$$

Assume (6) holds and $\left(s_{1}, \ldots, s_{6}\right) \neq\left(0,0,0,0, s_{5}, 0\right),\left(0,0,0,0,0, s_{6}\right)$. Then $\zeta_{\mathfrak{g}_{2}}\left(s_{1}, \ldots, s_{6}\right) \in$ $\mathcal{M P V}(w, d \leq 2,12)$, except for the two cases $\left(s_{1}, \ldots, s_{6}\right)=\left(0,0, s_{3}, 0,0,0\right),\left(0,0,0, s_{4}, 0,0\right)$ when $\zeta_{\mathfrak{g}_{2}}\left(s_{1}, \ldots, s_{6}\right) \in\langle\zeta(w-1), \zeta(w)\rangle_{\mathbb{Q}}$.

We are going to need the following combinatorial lemma to prove Theorem 1.1 . 
Lemma 1.2. Let $s, t$ be two positive integers. Let $x$ and $y$ be two non-zero real numbers such that $x+y \neq 0$. Then

$$
\frac{1}{x^{s} y^{t}}=\sum_{a=0}^{s-1}\left(\begin{array}{c}
t+a-1 \\
a
\end{array}\right) \frac{1}{x^{s-a}(x+y)^{t+a}}+\sum_{b=0}^{t-1}\left(\begin{array}{c}
s+b-1 \\
b
\end{array}\right) \frac{1}{y^{t-b}(x+y)^{s+b}} .
$$

Proof. Take $r=2$ in [15, Lemma 1].

\section{Proof of Theorem 1.1}

Before starting the proof we want to point out why it is necessary to exclude the two cases $\left(s_{1}, \ldots, s_{6}\right)=\left(0,0,0,0, s_{5}, 0\right),\left(0,0,0,0,0, s_{6}\right)$. Clearly

$$
\begin{aligned}
\zeta_{\mathfrak{g}_{2}}(0,0,0,0, s, 0) & =\sum_{m, n}^{\infty}\left(\frac{1}{(3(m+n))^{s}}+\frac{1}{(3(m+n)-1)^{s}}+\frac{1}{(3(m+n)-2)^{s}}\right) \\
& =\sum_{k=1}^{\infty}\left(\frac{k-1}{(3 k)^{s}}+\frac{k-1}{(3 k-1)^{s}}+\frac{k-1}{(3 k-2)^{s}}\right) \\
& =\frac{1}{3}\left(\zeta(s-1)-\sum_{k=1}^{\infty} \frac{3}{(3 k)^{s}}+\frac{2}{(3 k-1)^{s}}+\frac{1}{(3 k-2)^{s}}\right) \\
& =\frac{1}{3}\left(\zeta(s-1)-2 \zeta(s)-\sum_{k=1}^{\infty} \frac{1}{(3 k)^{s}}+\sum_{k=1}^{\infty} \frac{1}{(3 k-2)^{s}}\right) \\
& =\frac{1}{3} \zeta(s-1)-\frac{2}{3} \zeta(s)-\frac{1}{3^{s+1}} \zeta(s)-\frac{1}{3} \alpha(s)
\end{aligned}
$$

where $\alpha(s)=\sum_{k=1}^{\infty} 1 /(3 k-2)^{s}$. Fix a third root of unity $\nu=\exp (2 \pi i / 3)$. Then

$$
\alpha(s)=\frac{1}{3} \sum_{k=1}^{\infty} \frac{1+\nu^{k+2}+\nu^{2 k+4}}{k^{s}}=\frac{1}{3}\left(\zeta(s)+\nu^{2} L i_{s}(\nu)+\nu L i_{s}\left(\nu^{2}\right)\right)
$$

By numerical evidence we believe $\nu^{2} L i_{s}(\nu)+\nu L i_{s}\left(\nu^{2}\right) \notin \mathcal{M P V}(s, d \leq 2,12)$ for all positive integer $s$. For $s=1$ we have

$$
\nu^{2} L i_{1}(\nu)+\nu L i_{1}\left(\nu^{2}\right)=\frac{\sqrt{3} \pi}{6}+\frac{\log 3}{4} .
$$

Now the real values in $\mathcal{M P V}(1, d \leq 2,12)$ are generated by $\log (2 \cos (k \pi / 12)), k=0, \ldots, 5$, i.e., generated by

$$
\log 2, \log 3, \text { and } \log (\sqrt{3}+1),
$$

since $\cos (\pi / 12)=(\sqrt{6}+\sqrt{2}) / 4$ and $\cos (5 \pi / 12)=(\sqrt{6}-\sqrt{2}) / 4=\cos (\pi / 12) / 4$. Similar analysis can be applied to $\zeta_{\mathfrak{g}_{2}}\left(0,0,0,0,0, s_{6}\right)$.

Let's turn to the proof of Theorem 1.1] The domain of convergence (6) follows from the proof of [14, Prop. 2.1]. We now use step-by-step reductions to prove the rest of the theorem. First notice that if $\left(s_{1}, \ldots, s_{6}\right)=\left(0,0, s_{3}, 0,0,0\right),\left(0,0,0, s_{4}, 0,0\right)$ then $\zeta_{\mathfrak{g}_{2}}\left(s_{1}, \ldots, s_{6}\right)=$ 
$\zeta_{\mathfrak{s o}(5)}\left(s_{1}, \ldots, s_{4}\right)$ and the claim of the theorem is proved in [13, Case (i) and (iii.c)]. So in what follows we may assume that $\left(s_{1}, \ldots, s_{6}\right)$ is regular, i.e., $\left(s_{1}, \ldots, s_{6}\right)$ satisfies (6) and

$$
\left(s_{1}, \ldots, s_{6}\right) \neq\left(0,0, s_{3}, 0,0,0\right),\left(0,0,0, s_{4}, 0,0\right),\left(0,0,0,0, s_{5}, 0\right),\left(0,0,0,0,0, s_{6}\right) .
$$

\subsection{Reduction to eight cases of three nonzero variables by Lemma $\mathbf{1 . 2}$.}

2.1.1. General case to five nonzero variables. Taking $x=m$ and $y=n$ in Lemma 1.2 we see that

$$
\begin{aligned}
\zeta_{\mathfrak{g}_{2}}\left(s_{1}, \ldots, s_{6}\right) & =\sum_{a_{1}=0}^{s_{1}-1}\left(\begin{array}{c}
s_{2}+a_{1}-1 \\
a_{1}
\end{array}\right) \zeta_{\mathfrak{g}_{2}}\left(s_{1}-a_{1}, 0, s_{3}+s_{2}+a_{1}, s_{4}, s_{5}, s_{6}\right) \\
& +\sum_{a_{2}=0}^{s_{2}-1}\left(\begin{array}{c}
s_{1}+a_{2}-1 \\
a_{2}
\end{array}\right) \zeta_{\mathfrak{g}_{2}}\left(0, s_{2}-a_{2}, s_{3}+s_{1}+a_{2}, s_{4}, s_{5}, s_{6}\right) .
\end{aligned}
$$

2.1.2. Five nonzero variables to four nonzero variables. With $(x, y)=(m+3 n, 2 m+3 n)$ in Lemma 1.2 (since $x+y=3(m+2 n)$ ) we may reduce (77) to following two types of special values

$$
\begin{aligned}
\zeta_{\mathfrak{g}_{2}}\left(s_{1}, 0, s_{3}, s_{4}, s_{5}, 0\right) & =\sum_{m, n=1}^{\infty} \frac{1}{m^{s_{1}}(m+n)^{s_{3}}(m+2 n)^{s_{4}}(m+3 n)^{s_{5}}}, \\
\zeta_{\mathfrak{g}_{2}}\left(s_{1}, 0, s_{3}, s_{4}, 0, s_{6}\right) & =\sum_{m, n=1}^{\infty} \frac{1}{m^{s_{1}}(m+n)^{s_{3}}(m+2 n)^{s_{4}}(2 m+3 n)^{s_{6}}}
\end{aligned}
$$

and reduce (8) to

$$
\begin{aligned}
& \zeta_{\mathfrak{g}_{2}}\left(0, s_{2}, s_{3}, s_{4}, s_{5}, 0\right)=\sum_{m, n=1}^{\infty} \frac{1}{n^{s_{2}}(m+n)^{s_{3}}(m+2 n)^{s_{4}}(m+3 n)^{s_{5}}}, \\
& \zeta_{\mathfrak{g}_{2}}\left(0, s_{2}, s_{3}, s_{4}, 0, s_{6}\right)=\sum_{m, n=1}^{\infty} \frac{1}{n^{s_{2}}(m+n)^{s_{3}}(m+2 n)^{s_{4}}(2 m+3 n)^{s_{6}}},
\end{aligned}
$$

Indeed, we have

$$
\begin{aligned}
\zeta_{\mathfrak{g}_{2}}\left(s_{1}, 0, s_{3}, \ldots, s_{6}\right) & =\sum_{a_{5}=0}^{s_{5}-1}\left(\begin{array}{c}
s_{6}+a_{5}-1 \\
a_{5}
\end{array}\right) \frac{1}{3^{s_{6}+a_{5}}} \zeta_{\mathfrak{g}_{2}}\left(s_{1}, 0, s_{3}, s_{4}+s_{6}+a_{5}, s_{5}-a_{5}, 0\right) \\
& +\sum_{a_{6}=0}^{s_{6}-1}\left(\begin{array}{c}
s_{5}+a_{6}-1 \\
a_{6}
\end{array}\right) \frac{1}{3^{s_{5}+a_{6}}} \zeta_{\mathfrak{g}_{2}}\left(s_{1}, 0, s_{3}, s_{4}+s_{5}+a_{6}, 0, s_{6}-a_{6}\right)
\end{aligned}
$$

and similarly

$$
\begin{aligned}
\zeta_{\mathfrak{g}_{2}}\left(0, s_{2}, s_{3}, \ldots, s_{6}\right) & =\sum_{a_{5}=0}^{s_{5}-1}\left(\begin{array}{c}
s_{6}+a_{5}-1 \\
a_{5}
\end{array}\right) \frac{1}{3^{s_{6}+a_{5}}} \zeta_{\mathfrak{g}_{2}}\left(0, s_{2}, s_{3}, s_{4}+s_{6}+a_{5}, s_{5}-a_{5}, 0\right) \\
& +\sum_{a_{6}=0}^{s_{6}-1}\left(\begin{array}{c}
s_{5}+a_{6}-1 \\
a_{6}
\end{array}\right) \frac{1}{3^{s_{5}+a_{6}}} \zeta_{\mathfrak{g}_{2}}\left(0, s_{2}, s_{3}, s_{4}+s_{5}+a_{6}, 0, s_{6}-a_{6}\right) .
\end{aligned}
$$


2.1.3. Four nonzero variables to three nonzero variables. For any positive integers $a, b, s_{1}, s_{2}, s_{3}$ of which at least two of $s_{j}$ 's are nonzero such that $s_{2}+s_{3}>1$ and $s_{1}+s_{2}+s_{3}>2$ we define

$$
A_{a, b}\left(s_{1}, s_{2}, s_{3}\right):=\sum_{m, n=1}^{\infty} \frac{1}{m^{s_{1}}((a+1) m+b n)^{s_{2}}(a m+b n)^{s_{3}}} .
$$

We provide the details for reduction of (91) and leave that of (10)-(12) to the interested reader. Applying Lemma 1.2 with $(x, y)=(m+n, m+3 n)$ to (9) we can reduce it as follows:

$$
\begin{aligned}
\zeta_{\mathfrak{g}_{2}}\left(s_{1}, 0, s_{3}, s_{4}, s_{5}, 0\right) & =\sum_{a_{3}=0}^{s_{3}-1}\left(\begin{array}{c}
s_{5}+a_{3}-1 \\
a_{3}
\end{array}\right) \frac{1}{2^{s_{5}+a_{3}}} \zeta_{\mathfrak{g}_{2}}\left(s_{1}, 0, s_{3}-a_{3}, s_{4}+s_{5}+a_{3}, 0,0\right) \\
& +\sum_{a_{5}=0}^{s_{5}-1}\left(\begin{array}{c}
s_{3}+a_{5}-1 \\
a_{5}
\end{array}\right) \frac{1}{2^{s_{3}+a_{5}}} \zeta_{\mathfrak{g}_{2}}\left(s_{1}, 0,0, s_{4}+s_{3}+a_{5}, s_{5}-a_{5}, 0\right) \\
& =\sum_{a_{3}=0}^{s_{3}-1}\left(\begin{array}{c}
s_{5}+a_{3}-1 \\
a_{3}
\end{array}\right) 2^{s_{3}-s_{5}-2 a_{3}} A_{1,2}\left(s_{1}, s_{3}-a_{3}, s_{4}+s_{5}+a_{3}\right) \\
& +\sum_{a_{5}=0}^{s_{5}-1}\left(\begin{array}{c}
s_{3}+a_{5}-1 \\
a_{5}
\end{array}\right) 3^{s_{4}+s_{3}+a_{5}} 2^{s_{5}-s_{3}-2 a_{5}} A_{2,6}\left(s_{1}, s_{4}+s_{3}+a_{5}, s_{5}-a_{5}\right) .
\end{aligned}
$$

Applying Lemma 1.2 with $(x, y)=(m+n, m+3 n)$ to (11) we get

$$
\begin{aligned}
\zeta_{\mathfrak{g}_{2}}\left(0, s_{2}, s_{3}, s_{4}, s_{5}, 0\right) & =\sum_{a_{3}=0}^{s_{3}-1}\left(\begin{array}{c}
s_{5}+a_{3}-1 \\
a_{3}
\end{array}\right) \frac{1}{2^{s_{5}+a_{3}}} A_{1,1}\left(s_{2}, s_{4}+s_{5}+a_{3}, s_{3}-a_{3}\right) \\
& +\sum_{a_{5}=0}^{s_{5}-1}\left(\begin{array}{c}
s_{3}+a_{5}-1 \\
a_{5}
\end{array}\right) \frac{1}{2^{s_{3}+a_{5}}} A_{2,1}\left(s_{2}, s_{5}-a_{5}, s_{4}+s_{3}+a_{5}\right) .
\end{aligned}
$$

We may apply Lemma 1.2 with $(x, y)=(m+n, m+2 n)$ to (10) to get

$$
\begin{aligned}
\zeta_{\mathfrak{g}_{2}}\left(s_{1}, 0, s_{3}, s_{4}, 0, s_{6}\right) & =\sum_{a_{3}=0}^{s_{3}-1}\left(\begin{array}{c}
s_{4}+a_{3}-1 \\
a_{3}
\end{array}\right) 3^{s_{3}-a_{3}} A_{2,3}\left(s_{1}, s_{3}-a_{3}, s_{6}+s_{4}+a_{3}\right) \\
+ & \sum_{a_{4}=0}^{s_{4}-1}\left(\begin{array}{c}
s_{3}+a_{4}-1 \\
a_{4}
\end{array}\right) 3^{s_{4}-a_{4}} 2^{s_{6}+s_{3}+a_{4}} A_{3,6}\left(s_{1}, s_{6}+s_{3}+a_{4}, s_{4}-a_{4}\right) .
\end{aligned}
$$

and to (12) to get

$$
\begin{aligned}
\zeta_{\mathfrak{g}_{2}}\left(0, s_{2}, s_{3}, s_{4}, 0, s_{6}\right) & =\sum_{a_{3}=0}^{s_{3}-1}\left(\begin{array}{c}
s_{4}+a_{3}-1 \\
a_{3}
\end{array}\right) 2^{s_{3}-a_{3}} A_{2,2}\left(s_{2}, s_{6}+s_{4}+a_{3}, s_{3}-a_{3}\right) \\
& +\sum_{a_{4}=0}^{s_{4}-1}\left(\begin{array}{c}
s_{3}+a_{4}-1 \\
a_{4}
\end{array}\right) 2^{s_{4}-a_{4}} A_{3,2}\left(s_{2}, s_{4}-a_{4}, s_{6}+s_{3}+a_{4}\right) .
\end{aligned}
$$

To summarize we have reduced the general special values of $\zeta_{\mathfrak{g}_{2}}$ to the eight cases given by (14)-(21). Moreover, if we start with a regular value then after the above reduction steps we still get regular values. 
2.2. Computation of $A_{a, b}\left(s_{1}, s_{2}, s_{3}\right)$. Throughout this subsection for any positive integer $N$ let $\mu_{N}$ be a fixed primitive $N$-th root of unity. If we naïvely apply Lemma 1.2 with $x=(a+1) m+b n$ and $y=-a m-b n$ to (13) then we would get divergent series because of the convergence condition (5) of the multi-polylogs. Thus we need to modify it by calculating $A_{a, b}\left(s_{1}, s_{2}, s_{3}\right)$ as follows:

$$
\begin{aligned}
A_{a, b}\left(s_{1}, s_{2}, s_{3}\right) & =\sum_{m, n=1}^{\infty} \frac{(-1)^{s_{3}}}{m^{s_{1}}((a+1) m+b n)^{s_{2}}(-a m-b n)^{s_{3}}} \\
& =\sum_{a_{3}=0}^{s_{3}-2}\left(\begin{array}{c}
s_{2}+a_{3}-1 \\
a_{3}
\end{array}\right)(-1)^{a_{3}} B_{a, b}\left(s_{1}+s_{2}+a_{3}, s_{3}-a_{3}\right) \\
& +\sum_{a_{2}=0}^{s_{2}-2}\left(\begin{array}{c}
s_{3}+a_{2}-1 \\
a_{2}
\end{array}\right)(-1)^{s_{3}} B_{a+1, b}\left(s_{1}+s_{3}+a_{2}, s_{2}-a_{2}\right) \\
& +\frac{(-1)^{s_{3}}}{b}\left(\begin{array}{c}
s_{2}+s_{3}-2 \\
s_{3}-1
\end{array}\right)\left(C_{a, b}\left(s_{1}+s_{2}+s_{3}-1\right)+\lim _{M \rightarrow \infty} S_{a}^{(M)}\left(s_{1}+s_{2}+s_{3}-1\right)\right)
\end{aligned}
$$

where the functions $B, C$ and $S$ are defined as follows. For any positive integers $a, b, r$ and $s$ (with $s>1$ )

$$
\begin{aligned}
B_{a, b}(r, s):=\sum_{m, n=1}^{\infty} \frac{1}{m^{r}(a m+b n)^{s}} & =\frac{a^{r-1}}{b} \sum_{j=0}^{b-1} \sum_{k=0}^{a-1} \sum_{m, n=1}^{\infty} \frac{1}{m^{r}} \frac{\mu_{a}^{k m} \mu_{b}^{j n}}{(m+n)^{s}} \\
= & \frac{a^{r-1}}{b} \sum_{j=0}^{b-1} \sum_{k=0}^{a-1} L i_{s, r}\left(\mu_{b}^{j}, \mu_{a}^{k} \mu_{b}^{-j}\right) \in \mathcal{M P V}(r+s, 2, \operatorname{lcm}(a, b)) .
\end{aligned}
$$

where $\mathrm{lcm}$ denotes the lowest common multiple. For any positive integers $a, b$ and $s$ (with $s>1)$

$$
\begin{aligned}
C_{a, b}(s) & :=\sum_{j=1}^{b-1} \sum_{m, n=1}^{\infty} \frac{1}{m^{s}}\left(\frac{\mu_{b}^{j n}}{(a+1) m+n}-\frac{\mu_{b}^{j n}}{a m+n}\right) \\
& =\sum_{j=1}^{b-1}\left((a+1)^{s-1} \sum_{k=0}^{a} L i_{1, s}\left(\mu_{b}^{j}, \mu_{a+1}^{k} \mu_{b}^{-j}\right)-a^{s-1} \sum_{k=1}^{a} L i_{1, s}\left(\mu_{b}^{j}, \mu_{a}^{k} \mu_{b}^{-j}\right)\right) \\
& \in \mathcal{M P V}(s+1,2, \operatorname{lcm}(a, a+1, b)) .
\end{aligned}
$$


For any positive integers $a$ and $s$ (with $s>1$ )

$$
\begin{aligned}
S_{a}^{(M)}(s) & :=\sum_{m, n=1}^{M} \frac{1}{m^{s}}\left(\frac{1}{(a+1) m+n}-\frac{1}{a m+n}\right) \\
& =\sum_{m, n=1}^{M} \frac{1}{m^{s}}\left(\frac{1}{(a+1) m+n}-\frac{1}{a m+n}\right) \\
& =\sum_{m=1}^{M} \frac{1}{m^{s}}\left(\sum_{n=1+(a+1) m}^{M+(a+1) m}-\sum_{n=1+a m}^{M+a m}\right) \frac{1}{n} \\
& =\sum_{m=1}^{M} \frac{1}{m^{s}}\left(\sum_{n=M+a m+1}^{M+(a+1) m}+\sum_{n=1}^{a m}-\sum_{n=1}^{(a+1) m}\right) \frac{1}{n} .
\end{aligned}
$$

Noticing that $s>1$ and therefore

$$
\sum_{m=1}^{M} \frac{1}{m^{s}} \sum_{n=M+a m+1}^{M+(a+1) m} \frac{1}{n}<\sum_{m=1}^{M} \frac{1}{m^{s-1} M} \ll \frac{\log M}{M} \rightarrow 0 \quad \text { as } \quad M \rightarrow \infty
$$

we quickly see that

$$
\begin{aligned}
S_{a}(s) & :=\lim _{M \rightarrow \infty} S_{a}^{(M)}(s)=a^{s-1} \sum_{k=1}^{a} \sum_{m \geq n \geq 1} \frac{\mu_{a}^{k m}}{m^{s} n}-(a+1)^{s-1} \sum_{k=1}^{a+1} \sum_{m \geq n \geq 1} \frac{\mu_{a+1}^{k m}}{m^{s} n} \\
= & a^{s-1} \sum_{k=1}^{a}\left(L i_{s, 1}\left(\mu_{a}^{k}, 1\right)+L i_{s+1}\left(\mu_{a}^{k}\right)\right)-(a+1)^{s-1} \sum_{k=1}^{a+1}\left(L i_{s, 1}\left(\mu_{a+1}^{k}, 1\right)+L i_{s+1}\left(\mu_{a+1}^{k}\right)\right) .
\end{aligned}
$$

By the expressions for $B_{a, b}(r, s), C_{a, b}(s)$ and $S_{a}(s)$ we clearly have

$$
A_{a, b}\left(s_{1}, s_{2}, s_{3}\right) \in \mathcal{M P} \mathcal{V}\left(s_{1}+s_{2}+s_{3}, d \leq 2, \operatorname{lcm}(a, a+1, b)\right)
$$

Therefore Theorem 1.1 follows from (14)-(21) immediately. This concludes the proof of Theorem 1.1 . 


\section{Some Numerical Examples}

We remark that the twelfth root of unity $\mu_{12}$ appears essentially in the computation of (19). For example we have

$$
\begin{aligned}
\zeta_{\mathfrak{g}_{2}}(1,0,0,1,0,1) & \left.=\sum_{m, n=1}^{\infty} \frac{1}{m(m+2 n)(2 m+3 n)}=-C_{3,6}(2)-S_{3}(2)\right) \\
& =\sum_{j=1}^{5}\left(3 \sum_{k=1}^{3} L i_{1,2}\left(\mu_{6}^{j}, \mu_{3}^{k} \mu_{6}^{-j}\right)-4 \sum_{k=0}^{3} L i_{1,2}\left(\mu_{6}^{j}, \mu_{4}^{k} \mu_{6}^{-j}\right)\right) \\
& +4 \sum_{k=1}^{4}\left(L i_{2,1}\left(\mu_{4}^{k}, 1\right)+L i_{3}\left(\mu_{4}^{k}\right)\right)-3 \sum_{k=1}^{3}\left(L i_{2,1}\left(\mu_{3}^{k}, 1\right)+L i_{3}\left(\mu_{3}^{k}\right)\right) \\
& =0.29118204074051670279981404910049215137872186455887
\end{aligned}
$$$$
44495517845170396367225951404279842529727941799295
$$

with the error bounded by $10^{-100}$ using the computer program GiNac developed by Vollinga and Weinzierl [7]. With our current state of knowledge it is very difficult to prove rigorously that this value does not lie in $\mathcal{M P V}(3, d \leq 2, N)$ for any proper divisor $N$ of 12 . However, by assuming Grothendieck's period conjecture it might be possible to prove it by finding explicitly the bases of $\mathcal{M P V}(3, d \leq 2, N)$ using the ideas of [10, 11]. To illustrate this let us fix a third root of unity $\nu=\exp (2 \pi i / 3)$ as before and consider

$$
\begin{aligned}
\zeta_{\mathfrak{g}_{2}}(1,0,0,0,2,0)=\sum_{m, n=1}^{\infty} \frac{1}{m(m+3 n)^{2}} & =\frac{1}{3} \sum_{m, n=1}^{\infty} \frac{1+\nu^{n}+\nu^{2 n}}{m(m+n)^{2}} \\
& =\frac{1}{3}\left(\zeta(2,1)+L i_{2,1}\left(\nu, \nu^{2}\right)+L i_{2,1}\left(\nu^{2}, \nu\right)\right)
\end{aligned}
$$

which lies in the $\mathbb{Q}$-vector space $\mathcal{M P V}(3,3)$ generated by the special values of multi-polylog of weight three and level three. Assuming a variant of Grothendieck's period conjecture, Deligne [3] constructs explicitly a set of basis for $\mathcal{M P V}(w, N)$ and in particular predicts that $\operatorname{dim}_{\mathbb{Q}} \mathcal{M P V}(3,3)=8$. In [11] by using (regularized) double shuffle relations (RDS) we can explicitly construct the following basis of $\mathcal{M P V}(3,3)$ :

$$
\begin{array}{llll}
L i_{2,1}(\nu, 1), & L i_{1,1,1}(\nu, \nu, 1), & L i_{1,1,1}\left(\nu^{2}, 1, \nu^{2}\right), & L i_{1,1,1}\left(\nu^{2}, \nu^{2}, \nu^{2}\right) \\
L i_{2,1}(1, \nu), & L i_{1,1,1}(\nu, \nu, \nu), & L i_{1,1,1}\left(\nu^{2}, \nu^{2}, 1\right), & L i_{1,1,1}\left(\nu, \nu^{2}, \nu^{2}\right) .
\end{array}
$$

Further we can show by using RDS that

$$
\begin{gathered}
\zeta(3)=63 L_{1,1,1}(\nu, \nu, 1)+63 L_{1,1,1}\left(\nu^{2}, \nu^{2}, \nu^{2}\right)+18 L_{1,1,1}\left(\nu^{2}, 1, \nu^{2}\right)-126 L_{2,1}(\nu, 1) \\
-81 L_{1,1,1}\left(\nu^{2}, \nu^{2}, 1\right)-9 L_{1,1,1}(\nu, \nu, \nu)-54 L_{1,1,1}\left(\nu, \nu^{2}, \nu^{2}\right)-18 L_{2,1}(1, \nu),
\end{gathered}
$$


while

$$
\begin{aligned}
\zeta_{\mathfrak{g}_{2}}(1,0,0,0,2,0) & =52 L_{1,1,1}\left(\nu^{2}, \nu^{2}, 1\right)+84 L_{2,1}(\nu, 1)-\frac{112}{3} L_{1,1,1}\left(\nu^{2}, \nu^{2}, \nu^{2}\right)+\frac{8}{3} L_{1,1,1}(\nu, \nu, \nu) \\
& +\frac{116}{3} L_{1,1,1}\left(\nu, \nu^{2}, \nu^{2}\right)+12 L_{2,1}(1, \nu)-\frac{124}{3} L_{1,1,1}(\nu, \nu, 1)-\frac{44}{3} L_{1,1,1}\left(\nu^{2}, 1, \nu^{2}\right) .
\end{aligned}
$$

Clearly $\zeta_{\mathfrak{g}_{2}}(1,0,0,0,2,0)$ is not a rational multiple of $\zeta(3)$ (under the assumption of a variant of Grothendieck's period conjecture) since otherwise $\operatorname{dim}_{\mathbb{Q}} \mathcal{M P V}(3,3)<8$. Therefore $\zeta_{\mathfrak{g}_{2}}(1,0,0,0,1,0)$ cannot lie in $\mathcal{M P} \mathcal{V}(3,1)$ which is generated by $\zeta(3)=\zeta(2,1)$.

By applying our reduction process in the proof of Theorem 1.1 and then using GiNac we may also compute numerically

$$
\begin{aligned}
& \zeta_{\mathfrak{g}_{2}}(1,1,1,1,1,1)=0.01110008020419022277039637092289017833699879038194 \backslash \\
& 45086731059450527930767014636924683152705726916209 \text {. . }
\end{aligned}
$$

and verify directly that

$$
\sum_{m, n=1}^{300} \frac{1}{m n(m+n)(m+2 n)(m+3 n)(2 m+3 n)}=0.01110008013 \ldots
$$

Similarly

$$
\begin{aligned}
& \zeta_{\mathfrak{g}_{2}}(2,1,1,1,1,1)=0.00995272345287837349624059820619791150631359962925 \backslash \\
& 76058197642267125491591895578259027698791211370695 \text {. . } \\
& \zeta_{\mathfrak{g}_{2}}(1,2,1,1,1,1)=0.01051743558635248267821710084904837131835692774970 \backslash \\
& 58926903804614080083290282688685278848741106023759 \ldots \\
& \zeta_{\mathfrak{g}_{2}}(1,1,2,1,1,1)=0.00497203096318456908722247199264938834521998397322 \backslash \\
& 59104469026433274260043289411830973101381601191180 \ldots \\
& \zeta_{\mathfrak{g}_{2}}(1,1,1,2,1,1)=0.00334830993415208689505176677618470935993308677601 \backslash \\
& 07755998804241800305039265855252311030101487133354 \ldots \\
& \zeta_{\mathfrak{g}_{2}}(1,1,1,1,2,1)=0.00252989581107351464804145549755978591413008088242 \backslash \\
& 72342790902902770229510932678198294307546061097483 \ldots \\
& \zeta_{\mathfrak{g}_{2}}(1,1,1,1,1,2)=0.00199953266044219311834226765773032132006109764665 \backslash \\
& 46802961304161882193488113910506120491878470601712 \ldots
\end{aligned}
$$

Computation by Maple of the partial sums $\sum_{m, n=1}^{300}$ of the above values shows that they agree with each other for at least the first ten decimal digits. Finally, the most interesting special 
value is

$$
\zeta_{\mathfrak{g}_{2}}(2,2,2,2,2,2)=0.00007135906438752907355938750633729102655371125360 \backslash
$$$$
34722293695865502120914689101295543182083895277791 \ldots
$$

By the linear relation detection program PSLQ implemented with EZ-face [1] we find that

$$
\zeta_{\mathfrak{g}_{2}}(2,2,2,2,2,2) \stackrel{?}{=} \frac{2^{6} \cdot 5^{3} \cdot 23}{3^{6} \cdot 7^{2} \cdot 11 \cdot 13} \zeta(2)^{6}=\frac{5^{3} \cdot 23}{3^{12} \cdot 7^{2} \cdot 11 \cdot 13} \pi^{12}
$$

with the error bounded by $10^{-100}$. This is in agreement with the original discovery of Witten that for any positive integer $m \zeta_{\mathfrak{g}_{2}}\left(\{2 m\}_{6}\right)=c_{m} \pi^{12 m}$ for some $c_{m} \in \mathbb{Q}$. It might be possible to use the techniques developed in [4, 5] to find a closed formula for $c_{m}$ involving Bernoulli numbers.

Acknowledgement. The author would like to thank the Max-Planck-Institut für Mathematik for providing financial support during his sabbatical leave when this work was done. He also greatly benefited from several discussions with Andrey Levin, Don Zagier and Wadim Zudilin, especially Levin's suggestion to extend the results in [13, 14] to the exceptional Lie algebra $\mathfrak{g}_{2}$.

\section{REFERENCES}

[1] J. Borwein, P. Lisonek, and P. Irvine, An interface for evaluation of Euler sums. Available online at http://oldweb.cecm.sfu.ca/cgi-bin/EZFace/zetaform.cgi

[2] N. Bourbaki, Groupe et Algèbres de Lie, Chapitres 4, 5 et 6, Masson, Paris, 1981.

[3] P. Deligne, Le groupe fondamental de la $\mathbb{G}_{m}-\boldsymbol{\mu}_{N}$, unpublished manuscript.

[4] Y. Komori, K. Matsumoto and H. Tsumura, On Witten multiple zeta-functions associated with semisimple Lie algebras II. Preprint.

[5] Y. Komori, K. Matsumoto and H. Tsumura, On Witten multiple zeta-functions associated with semisimple Lie algebras III. Preprint.

[6] K. Matsumoto and H. Tsumura, On Witten multiple zeta-functions associated with semisimple Lie algebras I, Ann. Inst. Fourier 56 (2006), 1457-1504.

[7] J. Vollinga, S. Weinzierl, Numerical evaluation of multiple polylogarithms, arXiv:hep-ph/0410259

[8] E. Witten, On quantum gauge theories in two-dimensions. Commun. Math. Phys. 141(1) (1991), $153-209$.

[9] D. Zagier, Values of zeta function and their applications. Proc. of the First European Congress of Math. 2 (1994), 497-512.

[10] J. Zhao, Multiple polylogarithm values at roots of unity. C. R. Acad. Sci. Paris, Ser. I. 346 (2008), 10291032.

[11] J. Zhao, Standard relations of multiple polylogarithm values at roots of unity. Submitted.

[12] J. Zhao, Analytic continuation of multiple polylogarithms. Analysis Mathematica, 33 (2007), 301-323.

[13] J. Zhao, Alternating Euler sums and special values of Witten multiple zeta function attached to so(5), arxiv: 0903.0473

[14] J. Zhao and X. Zhou, Witten multiple zeta values attached to $\mathfrak{s l}(4)$. arxiv: 0903.2383

[15] X. Zhou and D.M. Bradley, On Mordell-Tornheim sums and multiple zeta values. Preprint, 2008. 